Ann. Biol. anim. Bioch. Biophys., I974, 14 (4-A), 709-720.

\title{
ACTION D'UNE FLORE MICROBIENNE QUI NE DÉCONJUGUE PAS LES SELS BILIAIRES SUR LA MORPHOLOGIE ET LE RENOUVELLEMENT CELLULAIRE DE LA MUQUEUSE DE L'INTESTIN GRELE DU RAT
}

\author{
J.-C. MESLIN, E. SACQUET* et P. RAIBAUD** \\ Station de Recherches de Nutrition, \\ Centre national de Recherches zootechniques, I. N. R. A., \\ 78350 Jouy en Josas \\ * Service des Animaux sans Germes, \\ Centre national de la Recherche scientifique, \\ 91190 Gif sur Yvette \\ ** Laboratoire d'Écologie microbienne, \\ Centre national de Recherches zootechniques, I. N.R. A., \\ 78350 Jouy en Josas
}

\section{RÉSUMÉ}

L'étude de la morphologie et du renouvellement cellulaire de la muqueuse de l'intestin grêle est effectuée d'une part chez des rats axéniques et des rats holoxéniques et d'autre part chez des rats gnotoxéniques dont la flore microbienne ne déconjugue pas les sels biliaires.

La profondeur des cryptes, la longueur des villosités, la localisation du front de marquage, le nombre de cellules épithéliales dans ces mêmes compartiments et l'index mitotique sont déterminés à trois niveaux de l'intestin grêle.

L'action de cette flore microbienne, moins complexe que celle des sujets holoxéniques, s'exprime par des caractéristiques morphologiques, une cellularité et une activité de renouvellement de l'épithélium intestinal semblables à celles des rats holoxéniques. Ceci montre que, dans des conditions physiologiques normales, l'acide cholique libre n'est pas le seul facteur responsable du renouvellement cellulaire plus rapide observé chez les animaux holoxéniques par rapport aux animaux axéniques.

La comparaison entre le rat axénique $\left(^{1}\right)$ et le rat holoxénique permet d'apprécier l'action qu'exerce la flore microbienne du tractus digestif sur la morphologie et le renouvellement cellulaire de la muqueuse de l'intestin grêle. Les études faites chez

(1) Nous utilisons la terminologie proposée par RAIBAUD et al. (I966). 
le Rat et la Souris ont porté sur les dimensions des villosités et des cryptes à divers niveaux de l'intestin grêle, sur la surface de l'épithélium (GORDON et BRUCKNERKardoss, I96i ; Mesi, in, Sacque't et GUenet, I973), sur la vitesse de son renouvellement (Abrams, Bauer, Sprinz, i963; Guenet et al., I970 ; GaljaARd et al., I972) et sur la durée des différentes phases du cycle cellulaire (LESHER et al., I964).

Dans une étude récente, RANKEN, WiLSON et BEALMER (I97I) montrent que l'administration à des souris axéniques d'acide cholique libre accélère le renouvellement de l'épithélium intestinal au niveau de l'iléon qui retrouve ainsi des valeurs égales à celles qu'il possède chez la souris holoxénique. Comme la formation d'acides biliaires libres à partir des acides conjugués à la taurine ou à la glycine est un phénomène bactérien, on peut penser que cette déconjugaison est un mécanisme par lequel la flore microbienne accélère, chez l'animal holoxénique, le renouvellement de l'épithélium intestinal. Cependant, il est difficile d'apprécier l'importance de cette activité parce que, selon qu'ils sont libres ou conjugués, les acides biliaires sont absorbés à des vitesses différentes (SchifF et al., 1972) en sorte que leur concentration respective au niveau de l'intestin grêle ne renseigne pas sur l'importance du flux des acides biliaires libres à travers la muqueuse.

Nous avons essayé de savoir si d'autres phénomènes que la déconjugaison bactérienne des sels biliaires n'étaient pas à l'origine d'une accélération du renouvellement épithélial chez l'animal holoxénique.

Comme une étude précédente a permis d'établir que l'ablation du volumineux cæcum chez le rat axénique modifie le renouvellement de l'épithélium intestinal (SACQUET et al., I97I), nous nous sommes proposé d'étudier l'action qu'exerce la présence d'une flore microbienne qui ne déconjugue pas les sels biliaires in vitro et in vivo, mais qui réduit le cæcum du rat axénique à des valeurs proches de celles qu'il possède chez le rat holoxénique (VAN HeIJ ENoOR't et al., I972).

\section{MÉTHODOLOGIE,}

Il a été nécessaire, pour mener à bien cette expérience, d'utiliser un régime alimentaire semisynthétique. En effet, il est facile d'obtenir des mélanges bactériens qui produisent une réduction importante du cæcum du rat axénique, à condition d'employer un tel régime (SAcQUET et al., 1973). Ceci nous a amené à abandonner le régime commercial utilisé dans les expériences précédentes. En conséquence, nous avons déterminé à nouveau les valeurs concernant la morphologie de la muqueuse intestinale qui avaient été établies avec le régime commercial complexe.

Nous avons d'autre part tenu compte du fait que le renouvellement de l'épithélium intestinal est un phénomène cellulaire et qu'il pouvait donc être judicieux de mesurer non seulement les dimensions des cryptes, des villosités, ainsi que la distance parcourue par les premières cellules marquées par la thymidine tritiée, mais aussi de dénombrer les cellules au niveau de ces divers compartiments.

Comme dans l'étude morphologique précédente (Mescrn et al., r973) les sujets sont des rats Fischer mâles. Nous avons utilisé 5 rats axéniques , 5 rats holoxéniques et 5 rats gnotoxéniques âgés de I 30 jours. Ces derniers sont obtenus en établissant dans le tractus digestif de rats axéniques les micro-organismes suivants :8 souches de streptocoques anaérobies facultatives, un streptocoque anaérobie strict, un Butyribacterium, une Ristella, une Veillonella, un Plectridium, une levure du genre Torulopsis.

Le régime alimentaire semi-synthétique a la composition pondérale suivante : caséine 220 , amidon de maïs $5^{80}$, cellulose $5^{\circ}$, huile de maïs 90 , supplément minéral et vitaminique 50 et eau 220.

Les techniques de prélèvement, de fixation de l'intestin, de marquage des noyaux par la 
thymidine tritiée et d'histoautoradiographie ont été précédemment décrites (GUENET et al., I970 ; MesLrn et al., 1973). En tenant compte du résultat des expériences précédentes et du fait qu'il est impossible d'effectuer des numérations cellulaires sur un grand nombre d'animaux, nous nous sommes limité à examiner des sujets sacrifiés 27 heures après l'injection de thymidine tritiée.

Nous déterminons dans les 3 lots d'animaux et aux trois niveaux, duodénum $=$ Io $\mathrm{p}$. Ioo, jéjunum $=50 \mathrm{p}$. I00, iléon $=90 \mathrm{p}$. 100 de la longueur totale de l'intestin grêle, les caractères suivants :

- Profondeur des cryptes de Lieberkühn sur des coupes passant par leur axe (ro cryptes par niveau et par animal) et nombre de cellules épithéliales de la base au collet des cryptes.

- Longueur des villosités, depuis le collet de la crypte jusqu'au sommet de la villosité, sur des villosités coupées suivant leur axe (Io villosités par niveau et par animal) et nombre de cellules épithéliales dans ce même compartiment.

Pour mesurer le renouvellement cellulaire, on détermine sur les mêmes préparations histologiques la position du front des cellules marquées 27 heures après l'injection de thymidine tritiée ainsi que le nombre de cellules épithéliales de la base de la crypte jusqu'à ce front. Ces données sont complétées par la détermination de l'index mitotique. Un des sujets axéniques n'ayant pas incorporé la thymidine tritiée, ces déterminations n'ont été effectuées que sur 4 animaux dans ce lot.

Toutes les valeurs mesurées sont représentées par leur moyenne arithmétique et l'écart-type de cette moyenne. La comparaison de ces moyennes a été faite selon le test $t$ de Student.

\section{RÉSULTATS}

Le tableau et les figures I, 2, 3 et 4 rendent compte des résultats obtenus.

A la lecture du tableau, il apparaît que les trois lots de rats ne diffèrent pas par leur poids vif ni par la longueur de leur intestin grêle mesurée après fixation. L,e

TABLEAU I

Poids vifs et longueurs d'intestin grêle des trois lots de rats Fischer

\begin{tabular}{l|c|c|c|c}
\hline \multicolumn{1}{c|}{ Type de rats } & $n$ & Poids (g) & Cæcum (g) & $\begin{array}{c}\text { Longueur } \\
\text { d'intestin grêle } \\
\text { fixé (cm) }\end{array}$ \\
\hline $\begin{array}{l}\text { Axéniques } \\
\text { Gnotoxéniques }\end{array}$ & 5 & $311 \pm 1,8$ & $20,7 \pm 0,56(\mathrm{~S})$ & $86,8 \pm 2,08$ \\
Holoxéniques & 5 & $313 \pm 5,0$ & $\mathbf{6 , 1 6 \pm 0 , 6 0}$ & $84 \pm 3,64$ \\
& & & & \\
\hline
\end{tabular}

$n$ : nombre d'observations.

moyenne \pm écart-type de la moyenne.

(S) : différence significative.

volumineux cæcum des sujets axéniques est significativement différent de celui des sujets gnotoxéniques et holoxéniques. Les micro-organismes introduits dans le tractus digestif des rats axéniques produisent une réduction considérable de leur cæcum qui n'atteint pas cependant chez les rats gnotoxéniques ainsi obtenus la valeur observée chez les animaux holoxéniques. 
Ires figures I et 2 représentent l'ensemble des résultats qui concernent les cryptes, les villosités et le front de marquage. Dans la figure I, ces données sont exprimées par leur dimension linéaire, et dans la figure 2 par le nombre de cellules épithéliales qui sont présentes dans chacun de ces compartiments.

\section{Profondeur des cryptes, nombre de cellules épithéliales de la base au collet des cryptes (fig. I et 2)}

Chez les sujets holoxéniques, la profondeur des cryptes (fig. I) ne varie pas du duodénum à l'iléon. Chez les sujets gnotoxéniques, seul le duodénum présente des valeurs plus élevées que le jéjunum et l'iléon. Par contre, dans le lot axénique, la profondeur moyenne des cryptes décroît du duodénum à l'iléon.

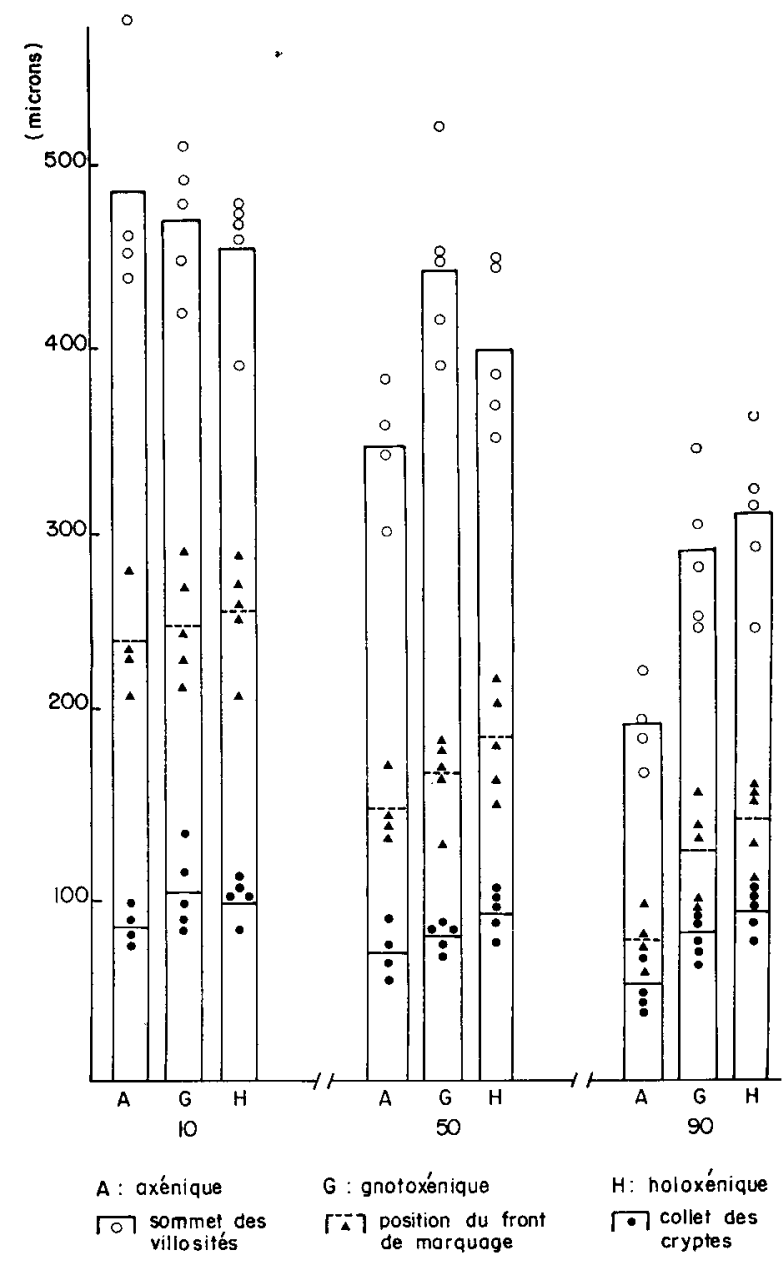

FrG. I. - Dimension des cryptes, des villosités et du compartiment des cellules marquées par la ${ }^{3}$ H-thymidine aux niveaux $10,50,90 \mathrm{p} .100$ de la longueur totale de l'intestin grêle

Les traits horizontaux représentent les valeurs moyennes, les points, triangles et cercles les valeurs individuelles 
Au niveau du duodénum, la présence d'une flore ne modifie pas la profondeur des cryptes (axéniques : $83 \pm 4$ microns ; gnotoxéniques : I02 \pm 9 microns ; holoxéniques : $98 \pm 5$ microns). Au niveau du jéjunum, une différence significative apparaît entre les axéniques et les holoxéniques. Les rats gnotoxéniques occupent une position intermédiaire entre les deux autres lots (axéniques $69 \pm 5$ microns ; gnotoxéniques : $78 \pm I$ microns ; holoxéniques : $9 \mathrm{I} \pm 5$ microns). Enfin, ces différences s'accusent encore au niveau de l'iléon ; la profondeur des cryptes chez les rats gnotoxéniques diffère significativement de celle des axéniques mais pas de celle des holoxéniques (axéniques : $50 \pm 7$ microns ; gnotoxéniques : $79 \pm 5$ microns ; holoxéniques : $9 \mathrm{I} \pm 5$ microns).

La distribution du nombre de cellules épithéliales (fig. 2) de la base au collet des cryptes est semblable à celle de la profondeur des cryptes, avec toutefois deux

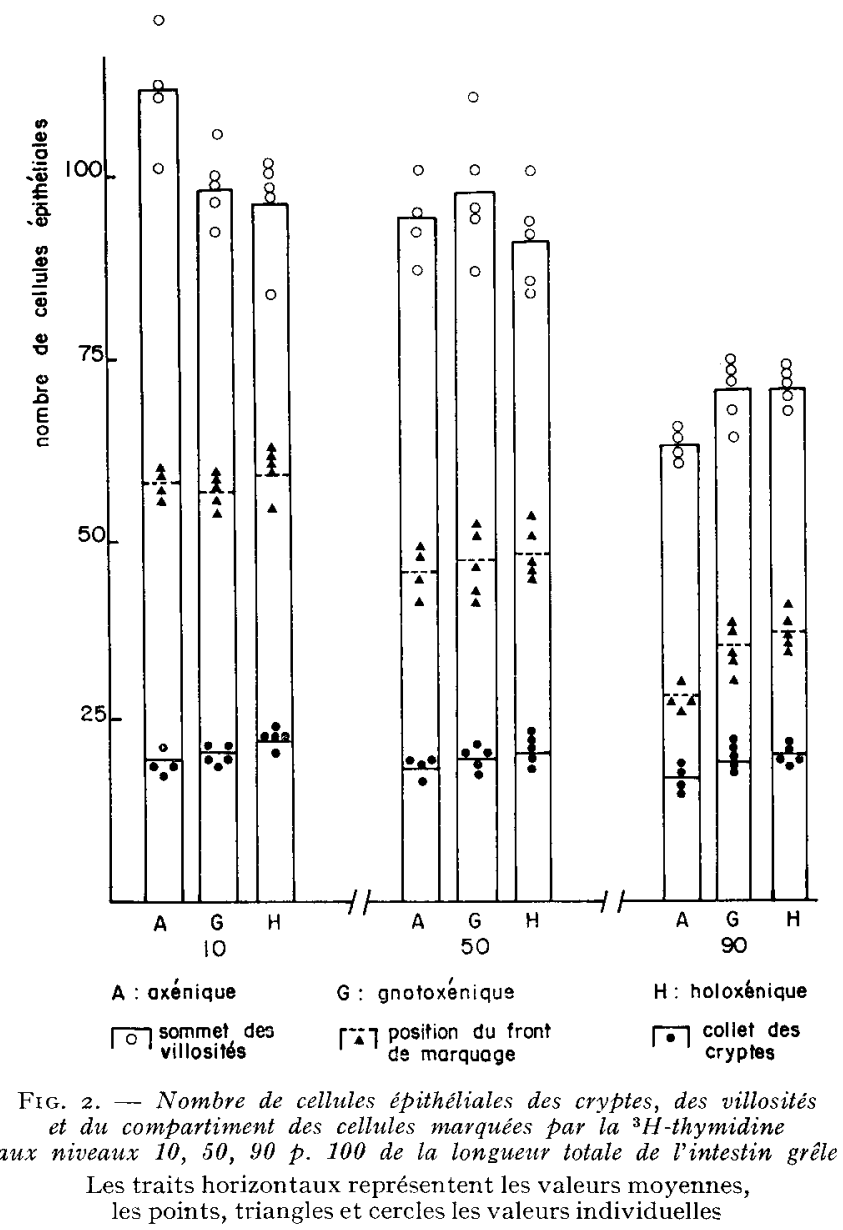

différences importantes : d'une part les différences ainsi observées entre Ies trois catégories de sujets sont plus faibles et les valeurs mesurées sont mieux groupées, et, d'autre part, au niveau du duodénum, le nombre de cellules épithéliales est signi- 
ficativement différent chez l'axénique et chez l'holoxénique alors que la profondeur ne l'est pas (duodénum, axéniques : I 9,5 上 0,7 cellules; gnotoxéniques : $2 \mathrm{I} \pm 0,2$ cellules; holoxéniques : $22,3 \pm 0,4$ cellules. Jéjunum, axéniques : I $8,2 \pm 0,5$ cellules; gnotoxéniques : I9,3 $\pm 0,4$ cellules ; holoxéniques : $2 \mathrm{I} \pm 0,3$ cellules. Iléon, axéniques : I6,9 $\pm 0,3$ cellules; gnotoxéniques : r9,4 $\pm 0,4$ cellules; holoxéniques : $20,3 \pm 0,2$ cellules).

\section{Longueur des villosités, nombre de cellules épithéliales au niveau des villosités}

La longueur des villosités (fig. I) décroît du duodénum jusqu'à l'iléon. Au niveau du jéjunum, les différences entre les rats axéniques et holoxéniques ne sont pas significatives ; par contre, les sujets gnotoxéniques qui diffèrent peu des holoxéniques présentent comparés aux sujets axéniques des valeurs significativement plus élevées (axéniques : $278 \pm \mathbf{I} 6$ microns ; gnotoxéniques : $367 \pm 22$ microns ; holoxéniques : $3 \mathrm{I} 2 \pm 24$ microns). Au niveau du duodénum et de l'iléon, la longueur des villosités est semblable chez les rats gnotoxéniques et holoxéniques. Au niveau du duodénum, elle tend à être plus courte chez les rats de ces deux groupes que chez les axéniques, mais la différence ne peut être affirmée (axéniques : $400 \pm 29$ microns ; gnotoxéniques: $369 \pm \mathrm{I} 4$ microns ; holoxéniques : $356 \pm \mathrm{I} 2$ microns). Au contraire, au niveau de l'iléon, comparée aux valeurs des sujets axéniques, elle est significativement plus grande (axéniques: $143 \pm 7$ microns ; gnotoxéniques : $209 \pm 13$ microns ; holoxéniques : $218 \pm \mathrm{I} 4$ microns).

Le nombre de cellules épithéliales présentes dans les villosités (fig. 2) varie chez les rats des divers groupes et aux différents niveaux de l'intestin grêle de la même manière que la longueur des villosités; cependant, il convient de remarquer que, au niveau du duodénum, les différences observées entre les axéniques et les deux autres lots sont plus accentuées que celles qui concernent la longueur des villosités (axéniques : $92 \pm 3$ cellules; gnotoxéniques : $76,8 \pm 2,2$ cellules; holoxéniques : $73,5 \pm 3$ cellules). Au niveau du jéjunum, les différences entre les trois lots d'animaux ne sont pas significatives (axéniques : 75,8 $\pm 2,3$ cellules; gnotoxéniques : 78,4 $\pm 3,8$ cellules; holoxéniques : $69,8 \pm 3$ cellules). Par contre, au niveau de l'iléon, les différences observées sont plus faibles que celles indiquées pour la longueur des villosités (axéniques : 45,4 $\pm \mathrm{I}$, I cellules ; gnotoxéniques : 50,6 \pm 2 cellules; holoxéniques : $49,8 \pm 0,8$ cellules).

\section{Position du front de marquage, nombre de cellules épithéliales dans le compartiment des cellules marquées (fig. $\mathrm{I}$ et 2 )}

A tous les niveaux de l'intestin grêle, la distance entre le front de marquage et la base des cryptes (fig. I) est moins grande chez les axéniques que chez les gnotoxéniques, et chez ceux-ci que chez les holoxéniques, mais les différences sont extrêmement faibles et non significatives au niveau du duodénum, (axéniques : 24I $\pm \mathrm{r}_{4}$ microns ; gnotoxéniques : $25 \mathrm{I} \pm \mathrm{I} 4$ microns; holoxéniques : $260 \pm \mathrm{r} 3,5$ microns), un peu plus marquées mais non significatives au niveau du jéjunum, (axéniques : I50 5 Io microns; gnotoxéniques: I70 \pm Io microns; holoxéniques : I87 \pm I3 microns), et significatives au niveau de l'iléon; à ce niveau, le compartiment des cellules marquées a une plus faible longueur chez les axéniques que chez les rats des 
deux autres lots qui ne diffèrent pas entre eux (axéniques : $77 \pm 7,5$ microns ; gnotoxéniques : I27 士 I3 microns; holoxéniques : I $43 \pm$ I I microns).

Le nombre de cellules épithéliales de la base de la crypte au front de marquage, que pour des raisons de simplicité de langage nous appellerons les cellules marquées (fig. 2), varie exactement de la même manière que la dimension du compartiment des cellules marquées, (duodénum : axéniques : 57,8 \pm I cellules ; gnotoxéniques : $56,5 \pm \mathrm{I}$ cellules ; holoxéniques : $59 \pm \mathrm{I}, 2$ cellules. Jéjunum, axéniques : $46 \pm \mathrm{I}, 6$ cellules; gnotoxéniques : $47 \pm 2$ cellules; holoxéniques : $48,6 \pm$ I,5 cellules). Au niveau de l'iléon, les valeurs sont significativement plus élevées en présence d'une flore, (axéniques : $28,7 \pm 0,5$ cellules ; gnotoxéniques : $35,4 \pm 1,5$ cellules ; holoxéniques : $37,7 \pm$ I cellules).

Le pourcentage des cellules marquées par rapport à l'ensemble des entérocytes est le suivant :

Duodénum, axéniques : 52 ; gnotoxéniques : 58 ; holoxéniques : 62 .

Jéjunum, axéniques : 49 , gnotoxéniques : 48 , holoxéniques : 54 .

Iléon, axéniques : 46 , gnotoxéniques : 50 ; holoxéniques : 54 .

\section{Index mitotique (fig. 3)}

Chez les sujets d'un même groupe, l'index mitotique varie peu d'un niveau à l'autre de l'intestin grêle : il tend à décrôttre légèrement du duodénum à l'iléon chez les sujets axéniques. A tous les niveaux, il existe une différence significative entre les

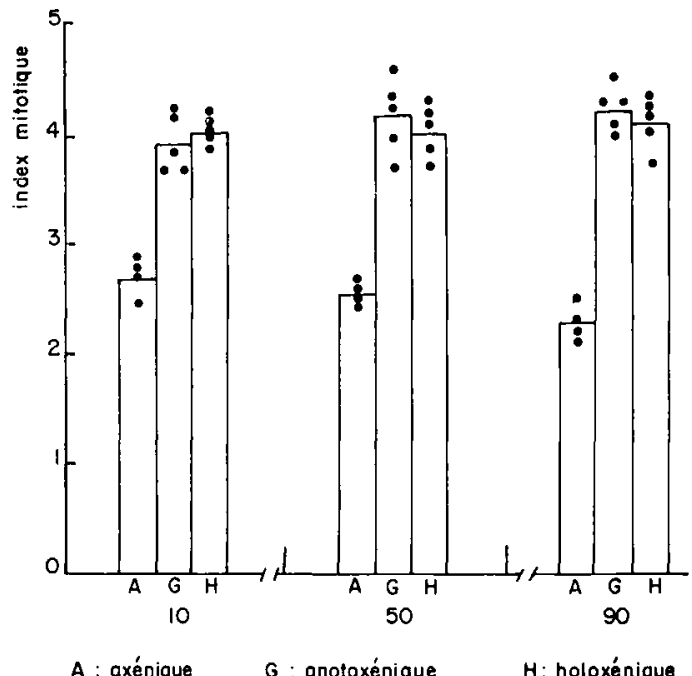

FIG. 3. - Index mitotique aux niveaux 10,50,90 p. 100 de la longueur totale de l'intestin grêle chez les trois lots de rats

Les traits horizontaux représentent les valeurs moyennes et les points les valeurs individuelles

sujets axéniques et ceux des groupes gnotoxéniques et holoxéniques. Chez ces deux derniers, cet index a la même valeur et est voisin de $4 \mathrm{p}$. Ioo alors que chez les axéniques, il varie de 2,8 au duodénum à 2,3 à l'iléon. 


\section{DISCUSSION}

La morphologie de l'épithélium intestinal et son renouvellement ont parfois été étudiés en mesurant les dimensions des différents compartiments : longueur des villosités, profondeur des cryptes, distance parcourue par les premières cellules marquées. Les résultats qui sont ainsi obtenus et ceux que l'on obtient en dénombrant les cellules de ces divers compartiments ne sont pas toujours équivalents parce que, dans le premier cas, ils dépendent de deux facteurs : le nombre de cellules et leurs dimensions, alors que, dans le second cas, ils ne dépendent que du premier de ces facteurs. Ia dispersion des résultats est dans l'ensemble plus grande lorsqu'est utilisée la première méthode, ce qui implique que les dimensions des cellules épithéliales, caractère qu'il serait difficile de mesurer sans erreur importante, sont plus variables que le nombre de ces cellules. Le diamètre moyen des cellules au niveau du jéjunum et de l'iléon est plus faible chez le rat axénique (jéjunum, axéniques : 3,6 microns; holoxéniques : 4,4 microns ; iléon, axéniques: 3,2 microns ; holoxéniques : 4,4 microns). Cette réduction du diamètre moyen des cellules au niveau de l'iléon du rat axénique est peut-être à rapprocher de l'observation que font Gustafsson et MAUnsbach (I97I) au niveau du cæcum chez le rat axénique. Chez celui-ci, ces auteurs observent en effet un plus grand nombre de noyaux par champ microscopique au niveau de cet organe, ce qui implique que le diamètre de ces cellules épithéliales soit diminué. Cette modification serait donc un phénomène qui prendrait naissance dès l'intestin grêle.

La plupart des caractéristiques morphologiques qui ont été précédemment observées chez les rats Fischer axéniques et holoxéniques (MESL.IN et al., I973) se retrouvent dans l'étude présente. Les villosités tendent à être plus longues au niveau du duodénum et au contraire plus courtes au niveau de l'iléon chez l'axénique comparé à l'holoxénique. Cet allongement au niveau du duodénum avait déjà été observé, bien que non mesuré, chez la Souris par LESHER et al. (1964). Toutefois, le régime alimentaire semble avoir une action très nette sur la longueur des villosités au niveau du duodénum. Si l'on compare les résultats de cette expérience à la précédente (MEsL,IN et al., I973), il apparaît que la longueur des villosités est beaucoup plus grande au niveau du duodénum chez les rats Fischer axéniques et holoxéniques qui recevaient un régime commercial que chez les rats Fischer de cette expérience-ci qui reçoivent un régime semi-synthétique (fig. 4). Au contraire, la nature de l'aliment semble n'exercer aucune action au niveau du jéjunum et de l'iléon qui ont des valeurs semblables dans ces deux expériences.

Nos observations concernant la profondeur des cryptes chez les sujets holoxéniques sont en accord avec les résultats d'ALTMANN et ENESCo (I967) qui n'observent aucune différence de cette profondeur aux divers niveaux de l'intestin grêle; par contre chez l'animal axénique, cette profondeur décroît du duodénum à l'iléon.

Les résultats des dénombrements cellulaires au niveau des villosités et des cryptes présentent des points d'accord et de désaccord avec ceux qui ont été effectués par d'autres auteurs : avec GALJAARD et al. (I972) qui ont limité leur étude au duodénum du rat, nous observons qu'au niveau des villosités, le nombre de cellules est augmenté chez le rat axénique comparé au rat holoxénique et le nombre de cellules que nous 
observons (axéniques : $92 \pm 3$ cellules; holoxéniques : 73,5 \pm 3 cellules) est très proche de celui qu'observent ces auteurs (axéniques : Io5 cellules; holoxéniques : 76 cellules). Mais par contre, nous sommes en désaccord avec eux quant au nombre de cellules comptées au niveau des cryptes (axéniques : I9,5 $\pm 0,7$ cellules ; holoxéniques : $22,3 \pm 0,4$ cellules. GALJAARD et al., axéniques : 32 cellules; holoxéniques : 3 I cellules). Il est possible que ce caractère dépende de la race et du régime alimentaire.

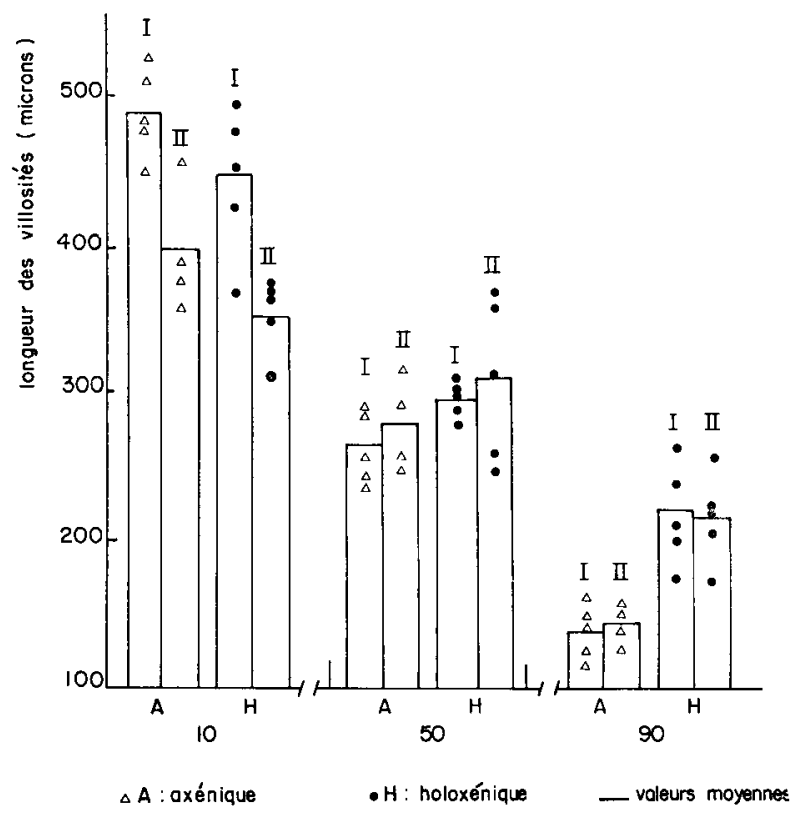

FIG. 4. - Longueur des villosités selon deux types d'expériences (I-II)

I : Régime commercial D. P.

II : Régime semi-synthétique S. N.

Aux niveaux ro, 5o, go p. roo de la longueur totale de l'intestin grêle

Le nombre de cellules que nous observons au niveau de l'iléon, aussi bien dans les villosités que dans les cryptes, varie, selon que les rats possèdent ou non une flore microbienne, dans le même sens que chez les souris étudiées par ABrams et al. (I963) : ce nombre augmente dans ces deux compartiments sous l'action de la flore (villosités: axéniques : $45,4 \pm \mathrm{I}$, I cellules; holoxéniques : $49,8 \pm 0,8$ cellules ; cryptes : axéniques : $16,9 \pm 0,3$ cellules; holoxéniques : $20,3 \pm 0,2$ cellules. ABrams et al. : villosités : axéniques : $36,0 \pm 0,8$ cellules; holoxéniques : $40,4 \pm 0,6$ cellules ; cryptes : axéniques: $\mathbf{I} 3,2 \pm 0, \mathrm{I}$ cellules; holoxéniques : I7,3 $\pm 0,3$ cellules ).

Pour tous ces caractères morphologiques : dimension des cryptes, des villosités et nombre de cellules épithéliales présentes dans ces deux compartiments, les rats gnotoxéniques se rapprochent très nettement des rats holoxéniques. Il a donc suffi de la présence de quelques souches microbiennes pour obtenir des modifications morphologiques comparables à celles qu'exerce la flore microbienne si complexe des rats holoxéniques. Cette observation conduit à penser qu'une étude étiologique de l'action de la flore microbienne sur la morphologie de l'intestin grêle est possible et, 
comme ces bactéries réduisent d'une manière importante le cæcum, on peut se demander si l'action qu'elles exercent au niveau de ces deux organes ne relève pas de mécanismes communs.

Si l'on considère la position du front de marquage 27 heures après l'injection de thymidine, celle-ci apparaît très différente de celle qui avait été obtenue dans l'expérience précédente. Cette expérience avait été faite à l'aide de rats qui recevaient un régime alimentaire de même composition mais qui étaient de race différente et qui n'étaient pas consanguins : des rats CF/Gif (GUENET et al., I97o). Dans cette expérience, la position du front de marquage avait été exprimée par le rapport entre la distance parcourue depuis le collet des cryptes par les premières cellules marquées par la thymidine tritiée et la longueur de la villosité. Ce rapport a dans les deux études des valeurs plus élevées chez le rat holoxénique que chez le rat axénique, ce qui suggère que le temps de renouvellement de l'épithélium est plus long chez le rat axénique que chez le rat holoxénique. Mais la différence ainsi observée entre rat axénique et rat holoxénique était beaucoup plus importante chez les rats CF de cette expérience que chez les rats Fischer de la présente expérience.

Les différences entre axéniques et holoxéniques apparaissent encore plus faibles quand on considère le nombre de cellules marquées par la thymidine tritiée plutôt que la position du front de marquage : aucune différence significative n'apparaît au niveau du duodénum, ce qui est conforme aux observations de GALJAARD et al. (I972), ni au niveau du jéjunum; seul l'iléon présente une différence significative.

La proportion de cellules épithéliales marquées est plus élevée au niveau du duodénum des rats holoxéniques qu'au niveau de celui des rats axéniques, mais ceci provient uniquement du plus petit nombre de cellules de l'épithélium chez le premier comparé au second et non pas d'un plus grand nombre de cellules marquées. Au niveau de l'iléon, cette proportion est aussi plus élevée chez l'holoxénique mais c'est en raison d'un plus grand nombre de cellules marquées et malgré un nombre de cellules épithéliales plus élevé que chez l'axénique.

Ceci suggère que la flore microbienne agit par deux mécanismes sur le renouvellement de l'épithélium : action sur le nombre de cellules de l'épithélium, action sur la position du front de marquage, et ces deux actions sont de valeur très inégale aux divers niveaux de l'intestin grêle.

Si la proportion des cellules de l'épithélium qui sont marquées par la thymidine tritiée présente, dans cette expérience, d'importantes différences par rapport à l'expérience antérieure, par contre, l'index mitotique garde des valeurs semblables dans les deux expériences. Cet index mitotique est à tous les niveaux de l'intestin grêle beaucoup plus faible chez le rat axénique et le nombre de cellules en mitose est relativement plus faible que ne le laisse prévoir le nombre de cellules marquées par la thymidine. Ceci est particulièrement net au niveau du duodénum où le nombre de cellules marquées est semblable et où le nombre de mitoses observées est 0,55 fois ce qu'il est chez l'holoxénique, donc presque deux fois plus faible. Ce désaccord ne peut s'expliquer que de deux manières : ou bien le mode de multiplication cellulaire est modifié par la présence d'une flore, par exemple présence de cellules filles qui se diviseraient une fois chez l'axénique et qui ne se diviseraient pas chez l'holoxénique, ce qui exigerait de la part de la cellule souche qu'elle se divise deux fois moins, ou bien le mode de multiplication est le même mais le temps de la mitose par rapport au temps du cycle cellulaire est deux fois plus court. LESHER et al. (I964) qui ont 
étudié les différentes phases du cycle cellulaire attirent l'attention sur une augmentation de la durée des phases $\mathrm{GI}$ et $\mathrm{S}$ chez les sujets axéniques mais n'indiquent pas s'il y a une modification du temps de la mitose.

Chez les sujets gnotoxéniques, le nombre de cellules épithéliales marquées et l'index mitotique ont la même valeur que chez les rats holoxéniques. Comme la flore microbienne de ces sujets ne produit pas d'acide cholique libre, il apparait que la déconjugaison des sels biliaires n'est pas la seule modification chimique par laquelle la flore microbienne accélère le renouvellement cellulaire de l'épithélium, et il y a lieu de s’interroger sur l'importance véritable de cette action.

Rę̧u pour publication en avril 1974.

\section{REMERCIEMENTS}

Ce travail a bénéficié d'une aide financière dans le cadre d'un contrat d'action thématique programmée du Centre national de la Recherche scientifique (thème : Écophysiologie des Régulations) $n^{\circ} 4602$.

\section{SUMMARY}

ACTION OF A MICROBIAL FLORA, WHICH DOES NOT DECONJUGATE BILE SALTS, ON THE MORPHOLOGY AND CELL RENEWAL, OF RAT SMALI, INTESTINAL MUCOSA

Study of the morphology and cell renewal of the small intestinal mucosa is carried out on axenic and holoxenic rats and on gnotoxenic rats having a microbial flora which does not deconjugate bile salts.

The depth of crypts, length of villi, localisation of labelling front, number of epithelial cells in these same compartments, and the mitotic index are determined at three levels in the small intestine.

The action of this microbial flora, which is less complex than that of holoxenic subjects, is expressed by morphological characteristics, cell number and renewal activity of the intestinal epithelium similar to those of holoxenic rats. This shows that in normal physiological conditions, free cholic acid is not the only factor responsible for the increased renewal of cells observed in holoxenic animals, as compared to axenic animals.

\section{RÉFÉRENCES BIBLIOGRAPHIQUES}

Abrams G. D., Baver H., Sprinz H., ig63. Influence of the normal flora on mucosal morphology and cellular renewal in the ileum. A comparison of germ-free and conventional mice. Lab. Invest., 12, $355-364$.

Altmann G. G., Enesco M., 1967. Cell number as a measure of distribution and renewal of epithelial cells in the small intestine of growing and adult rats. Am. J. Anat., 121, 319-336.

Galjaard H., Van der Meer-Fieggen W., Giesen J., i972. Feedback control by functional villus cells on cell proliferation and maturation in intestinal epithelium. Exp. Cell. Res., 73, 197-207.

Gordon H. A., Bruckner-Kardoss E., I96r. Effect of normal microbial flora on intestinal surface area. Amer. J. Physiol., 201, I75-I78.

Guenet J. L., Sacquet E., Gueneau G., Meslin J. C., i97o. Action de la microflore totale du ra sur l'activité mitotique des cryptes de Lieberkühn. C. R. Acxd. Sc., 270, 3087-309o. 
Gustafsson B. E., Maunsbach A. B., I97I. Ultrastructure of the enlarged cecum in germfree rats. Z. Zellforsch., 120, 555-578.

Lesher S., Walburg H. E., Sacher G. A., 1964. Generation cycle in the duodenal crypt cells of germ-free and conventional mice. Nature, 202, 884-886.

Meslin J. C., Sacouet E., Guenet J. L., 1973. Action de la flore bactérienne sur la morphologie et la surface de la muqueuse de l'intestin grêle du rat. Ann. Biol. anim. Biochim. Biophys., 13, $203-214$.

Raibaud P., Dickinson A. B., Sacquet E., Charlier H., Mocquot G., ig66. La microflore du tube digestif du rat. IV. Implantation contrôlée chez le rat gnotobiotique de différents genres microbiens isolés du rat conventionnel. Ann. Inst. Pasteur, 111, 193-210.

RANKen R., Wilson R., Bealmer P. M., I97x. Increased turnover of intestinal mucosal cells of germfree mice induced by cholic acid. Proc. Soc. Exp. Biol. Méd., 138, 270-272.

Sacquet E., Guenet J. L., Garnier H., Meslin J. C., ig7i. Influence de deux modifications chirurgicales de l'intestin grêle sur le renouvellement des entérocytes du rat : la formation d'une anse aveugle chez le rat holoxénique. L'ablation du cæcum chez le rat axénique. C. $R$. Acad, Sc., 272,84I-844.

Sacouet E., Lachkar M., Mathis C., Raibaud P., 1973. Cecal reduction in "gnotoxenic "rats. In Germfree research. Biological effect of gnotobiotic environments. Proceedings of the IVth International Symposium on Germfree Research. New Orleans. Louisiana. April 1972. Section XI : 545-552. J. B. HENEGHAN éd., Academic Press.

Schiff E. R., Small N. C., Dietschy J. M., 1972. Characterisation of the kinetics of the passive and active transport mechanisms for bile acid absorption in the small intestine and colon of the rat. J. Clin. Invest., 51, 1351-1362.

Van Heijenoort Y., Sacouet E., Raibaud P., Demarne Y., Mathis C., ig72. Action de la flore microbienne sur le contenu de l'intestin grêle du rat en acides biliaires. C. R. Acad. Sc., 275, 27 I-274. 